\title{
La lectura de palabras: la influencia del procesamiento fonológico y del método lectoescritor
}

\author{
Word Reading: the influence of phonological awareness and \\ teaching methods
}

\author{
Sara Isabel Rendón Romero \\ Eduardo García Jiménez \\ Macarena Navarro Pablo \\ Universidad de Sevilla
}

Recibido: 09/10/2018

Aceptado: 30/11/2018

\begin{abstract}
Phonological awareness and teaching methods implemented in the classroom can influence the development of early reading skills. Specifically, this paper aims at identifying which specific linguistic factors influence reading skills and how the context of classroom instruction can affect this learning process. Data have been collected from a sample composed of children belonging to the last year of Childhood Education of two different schools in Seville (State and Private schools) following a quantitative and qualitative methodology. On the one hand, tests related to the students' level of phonological awareness, speed naming and reading comprehension were carried out. On the other hand, interviews and observations to the teachers were conducted in order to get a deeper understanding of their teaching methods, activities and resources used inside the classroom. Results confirm that phonological awareness, letter and pseudo-word reading are strong predictors of word reading. Furthermore, students with a higher level of phonological awareness and reading have received formal instruction through a mixed teaching method combining the phonic and global method. It is evident that these students present a more advanced level of automation. In conclusion, this specific mixed teaching method undoubtedly favours children's reading development.
\end{abstract}

KEY WORDS: Teaching methods, phonological awareness, learning processes, reading, early literacy.

\section{RESUMEN}

La conciencia fonológica y el método de lectura usado en el aula pueden influir en el desarrollo de habilidades lectoras en edades tempranas. Este estudio tiene como objetivos principales determinar qué factores lingüísticos pueden influir en el aprendizaje lector y conocer el papel en que dicho aprendizaje juega el contexto de instrucción. Se han recogido datos a través de una metodología cuantitativa y cualitativa en una muestra compuesta por el alumnado del segundo ciclo de Educación Infantil de dos centros educativos de la provincia de Sevilla, uno público y otro privado. Se han realizado pruebas sobre el nivel de procesamiento fonológico, velocidad en denominación y nivel de lectura del alumnado participante, además de observaciones y entrevistas al profesorado para conocer su método de enseñanza de lectura, así como las actividades y los recursos utilizados en el aula. Los resultados muestran que el procesamiento fonológico y la lectura de letras y pseudopalabras son buenos predictores de la lectura de palabras. Además, los discentes que presentan un mayor nivel de conciencia fonológica y de lectura y han recibido instrucción formal, a partir de un método combinado fónico y global, muestran un grado más avanzado de automatización lectora. Concluimos que este método combinado favorece el desarrollo lector del alumnado.

PALABRAS CLAVE: Método de enseñanza, procesamiento fonológico, proceso de lectura, lectura, alfabetización temprana.

Dirección de correspondencia:

Sara Isabel Rendón Romero, Universidad de Sevilla, Facultad de Ciencias de la Educación. E-mail: sarrenrom@alum.us.es. ORCID: 0000-0002-1150-7822.

Eduardo García Jiménez, Universidad de Sevilla, Departamento de Métodos de Investigación y Diagnóstico en Educación. Facultad de Ciencias de la Educación. ORCID: 0000-0002-5885-8267.

Macarena Navarro Pablo, Universidad de Sevilla, Departamento de Didáctica de la Lengua y la Literatura y Filologías Integradas. Facultad de Ciencias de la Educación. ORCID: 0000-0003-1954-0851 


\section{Introducción}

En la enseñanza de la lectoescritura, tal y como indica Cuetos (2008), ya en la década de los sesenta, se plantea un debate que se ha mantenido en el contexto educativo de la enseñanza de la lectura. Se trata de la elección del método de lectura y especialmente la decisión sobre cuál debe ser la unidad de partida en su enseñanza: "la identificación de la letra o el reconocimiento de palabras globalmente a través de sus formas gráficas." (Cuetos, 2008, p. 32). En este trabajo se ha optado por seguir la propuesta de Cuetos de apoyar la importancia del reconocimiento previo de las letras. Este autor tiene en cuenta las teorías del Procesamiento Distribuido en Paralelo (PDP, McClelland, 1987) así como los niveles que existen en cuanto a la identificación de las palabras (nivel de rasgos, nivel de letras y nivel de palabras). A partir de estas propuestas, en la enseñanza de la lectura que tiene lugar en los primeros cursos escolares, los esfuerzos se centrarían en los procesos de decodificación que ayudan al alumnado a identificar las letras para poder leerlas. Para ello, en esos procesos es necesario que el discente adquiera un determinado nivel de conciencia fonológica.

La presente investigación trata de analizar los factores que pueden influir en la lectura temprana en la primera lengua, en este caso el español. Por una parte, tratamos de determinar el nivel de procesamiento fonológico (PF), la velocidad en denominación (VD) y el nivel de lectura de letras (LL), palabras (LP) y pseudopalabras (LPP) del alumnado. Por otra parte, estudiamos las relaciones de los resultados obtenidos por el alumnado y el método lectoescritor, los recursos y las actividades utilizadas en clase por el profesorado.

\section{Fundamentación teórica}

\subsection{El procesamiento fonológico}

El análisis de la conciencia fonológica del alumnado, caracterizada por la capacidad de reconocer sonidos para poder relacionar el fonema (la representación del sonido) con el grafema (la representación de la letra), resulta esencial y junto a otros aspectos lingüísticos puede contribuir al desarrollo de habilidades lectoras. La conciencia fonológica se desarrolla por fases dependiendo de las unidades que se manipulan, así se distingue entre conciencia silábica, conciencia intrasilábica y conciencia fonémica.

Los estudios relacionados con el proceso de aprendizaje de la lectura que señalan la existencia de una estrecha relación entre la conciencia fonológica y el proceso de lectura son numerosos. Concretamente Jiménez y Ortiz (2000) observaron la relación de la conciencia fonológica con la lectura de palabras y de pseudopalabras. Jiménez y Haro (1995) confirmaron la misma idea en torno a la conciencia silábica. Estos autores observaron que los discentes de su estudio eran capaces de leer sílabas sin conocer el alfabeto completo. En estudios posteriores, Jiménez y Ortiz (2007) señalaron que la decodificación de palabras escritas es parte fundamental en el proceso de lectura y la conciencia fonológica facilita dicho proceso.

Otros autores conceden aún más importancia al proceso de reconocimiento de las letras, dado que, además, le atribuyen la capacidad de predicción de la lectura. Así, en el estudio realizado por Ehri (1987) ya se demostraba que el reconocimiento de letras es uno de los mejores predictores de la lectura. Este autor comprobaba que cuando el alumnado recibía instrucción sobre la capacidad de deletrear aprendían a leer con mayor eficiencia ya que les permitía segmentar la pronunciación en sus respectivos constituyentes fonéticos.

En la actualidad, los estudios relacionados con la conciencia fonológica y el reconocimiento de palabras, además de identificar la relación entre la conciencia fonológica y la lectura (Edyburn et al., 2017; Hill, 2017), destacan la capacidad de la conciencia fonológica para predecir el rendimiento lector (Batson-Magnuson, 2017; Saracho, 2017, Biscoe Kenner, Patton Terry, Friehling y Namy, 2017;). A modo de ejemplo, Bellocchi, Tobia y Bonifacci (2017) han estudiado la posibilidad de predecir la exactitud en la lectura en el alumnado monolingüe. Por su parte, Suárez-Coalla, Garcíade-Castro y Cuetos (2013) han señalado la capacidad predictora del procesamiento fonológico en los siguientes términos: “(...) luego el procesamiento fonológico, especialmente la $\mathrm{CF}$, aparece como un 
importante predictor de la exactitud, (...) Las habilidades de procesamiento fonológico, (...), se revelaron como mejores predictores de la exactitud en lectura y escritura." (p. 77)

Con objeto de poder seleccionar los predictores más idóneos para nuestro estudio hemos tenido en cuenta dos variables importantes: la edad o momento de desarrollo del aprendizaje y la instrucción que recibe el discente. Jiménez y Artiles (2001) indicaban que no se debe fijar una edad cronológica para iniciar la lectura, sino que el profesor debería usar pruebas para conocer la madurez de cada uno de sus estudiantes y adaptar las actividades de manera individual. En este sentido, para determinar los mejores predictores debería considerarse la variable edad combinada con las características individuales y contextuales del alumnado. Junto a esta variable es también importante tener en cuenta la instrucción que se proporciona. En este sentido, algunos autores (Pinto, Bigozzi, Vezzani y Tarchi, 2016; Casillas y Goikoetxea, 2007; Márquez, 2013; De-La-Calle Cabrera, 2017) han estudiado la relación entre la alfabetización formal y el desarrollo de las habilidades lectoras tempranas. De igual modo, Schaars, Segers y Verhoeven (2017) encontraron que los precursores de la alfabetización temprana y la velocidad en denominación funcionan como predictores desde los primeros meses de desarrollo de la lectura de palabras. También, Parrilla, Kirby y McQuarrie (2004) observaron que la velocidad en denominación contribuía a predecir la variedad individual que existe en la lectura en el período del jardín de infancia. Sin embargo, con posterioridad a esta etapa (ya en Educación Primaria) la velocidad en denominación pierde capacidad de predicción y la conciencia fonológica se convierte en un predictor más consistente.

En lo que respecta a las diferencias individuales y al análisis de la conciencia fonológica es necesario tener en cuenta que la lectura en cada discente se desarrolla de una manera diferente, de tal forma que no todos avanzan en el mismo orden por las tres etapas propuestas por Frith (1986). Según este autor, la lectura comienza por la etapa logográfica, donde el alumnado empieza a identificar las palabras más frecuentes de su vida diaria. En este caso, Frith (1986) mencionaba que el alumnado empieza a identificar normalmente la primera letra de aquellas palabras que le resultan más familiares. A continuación, la lectura entra en una etapa donde la enseñanza formal es necesaria para que el discente comprenda y establezca la relación entre la grafía y el fonema. Ésta es la etapa alfabética. Por último, en la denominada etapa ortográfica, las dos fases anteriores se automatizan dando paso a una lectura más fluida. Es en esta etapa cuando el discente comienza a detectar la morfología de las palabras. Estas etapas permitirían, según Frith (1986), conocer el momento en el que se encuentra el desarrollo lector del discente.

Una vez que el alumnado va adquiriendo un mayor conocimiento fonológico y avanza en su proceso de decodificación, la lectura de grafemas, a través de una ruta subléxica, empieza a combinarse con la lectura de palabras a través de una ruta léxica. Así, Coltheart (1985) define el modelo de doble ruta como directa e indirecta o léxica y subléxica que, tal y como señala Cuetos (2008), es el modelo más conocido de reconocimiento de palabras. El autor lo describe de la siguiente forma:

De acuerdo con este modelo, existen dos vías para llegar desde la palabra escrita al significado y/o pronunciación: la vía léxica, que nos permite leer la palabra atendiendo directamente a (...) léxico visual, y la vía subléxica, que nos permite leer las palabras a través de la transformación de las letras en sus correspondientes sonidos, (...). (p. 43).

\subsection{Contexto de instrucción}

El tipo de instrucción que se lleve a cabo en las aulas resulta clave en el desarrollo del proceso lector. Los estudios realizados nos han mostrado a lo largo del tiempo que existen dos enfoques metodológicos preponderantes para la enseñanza de la lectura que parten de dos visiones diferentes sobre la misma. Guzmán-Simón, Navarro-Pablo y García-Jiménez (2015) hacen una descripción detallada de los principales métodos de enseñanza de la lectura y la tipología de actividades que los caracterizan. Por un lado, encontramos los métodos ascendentes o sintéticos cuya enseñanza comienza con el fonema, la grafía y la sílaba, concluyendo con la enseñanza de palabras, frases y 
textos. Estos métodos pueden ser implementados de manera alfabética, relacionando el nombre de la letra con la imagen de su grafía; fonética, relacionando el sonido con la grafía; o silábica, comenzando a partir del reconocimiento de las sílabas. Por el contrario, los métodos descendentes o analíticos parten de la unidad mínima de significado, la palabra, después la oración o texto para concluir con el aprendizaje de la sílaba, grafía y fonema. En este tipo de métodos distinguimos también tres modalidades: léxicos, donde se parte de las palabras que se ponen en relación con los dibujos; globales, que parten de las frases o textos y se relacionan con la experiencia del alumnado desarrollando la memoria visual y auditiva; o integrales que combinan los dos métodos anteriores.

Distinguiendo entre los distintos enfoques metodológicos, el método ascendente fónico se caracteriza por ser más rápido, dinámico y eficaz en el aprendizaje. Ello es debido a que al relacionar el fonema con la grafía el alumnado adquiere directamente la conciencia del fonema y de la grafía con mayor facilidad. De este modo, las habilidades lectoras del alumnado pueden mejorar notablemente (Cuetos, 2008; Jiménez et al., 2007; Schoaga, Akintola y Isiwat OKPOR, 2017). Además, Jiménez et al. (2001) indicaban que este tipo de método podrá proporcionar más autonomía en el aprendizaje del alumnado, ya que éste adquiere un conocimiento que le permitirá aplicar en cualquier lectura familiar y no familiar. A favor de los métodos descendentes, Jiménez et al. (2001) comentaban que dichos métodos analíticos respetan el interés y la necesidad del alumnado trabajando la creatividad en las aulas.

En lo que respecta a las actividades que se realizan en el aula y los recursos de los que dispone el profesorado, la investigación realizada por Lera Rodríguez (2007) en la provincia de Sevilla describe las prácticas docentes en los siguientes términos:

[El profesorado] se dedicaba sobre todo a informar al alumnado y supervisar su trabajo, por lo que las relaciones eran mayoritariamente con todo el grupo y con niños individuales (para comprobar su trabajo). El alumnado permanecía la mayor parte del tiempo sentado en pequeños grupos, haciendo sus tareas de manera individual. Estas tareas solían ser de motricidad fina (dibujar, escribir, repasar, picar, recortar), altamente cerradas y estructuradas, algunas de lenguaje (lectura de cuentos, explicación de fenómenos, etc.) y un poco de creatividad, música, de motricidad gruesa, con porcentajes que no superaban el 5\%. (p. 310).

Sin embargo, la literatura especializada señala cómo el aula debería conseguir un clima positivo haciendo uso de actividades basadas en experiencias reales, en el discurso diario y en las interacciones que tendrían como objetivo potenciar las habilidades comunicativas y lingüísticas (Beecher y Makin, 2002; Verhoeven, 2005; Bigas, 2002; Norling, Sandberg y Almqvist, 2015). Además, de acuerdo con Englezou y Fragkouli (2014), si dichas actividades se basan en la lectura en voz alta, en juegos, canciones y rimas beneficiarán no solo la motivación del alumnado sino el desarrollo de la conciencia fonológica y el desarrollo conceptual del lenguaje. Para ello, además, será importante el uso de materiales adecuados que potencien estas oportunidades de interacción real y desarrollo del alumnado (Fons, 2002). Montessori (2006) ya en 1907 y, posteriormente, Freinet en 1974, nos hablaban de atender las necesidades de los discentes a través de un aprendizaje natural basado en la experiencia real del mundo a través de la observación y experimentación ya que, así, se potencia la organización y autonomía del alumnado. En esta misma línea, Teberosky (2003) propuso que los alumnos escribieran atendiendo a sus necesidades de comunicación, y en "situaciones de interacción cotidiana" (p. 25), utilizando para ello la escritura de cartas, noticias, recetas de cocinas.... Esta es la escritura, tal y como indicaba Ferreiro (2002), a la que los niños están expuestos continuamente fuera de la escuela, siendo, así, un "objeto social, con existencia social" (p. 25), una "información lingüística" parecida al aprendizaje del lenguaje oral que también debería estar contextualizada para facilitar dicho aprendizaje. 


\section{Diseño y metodología}

Este estudio plantea conocer cuáles son los factores lingüísticos y no lingüísticos que proporcionan más información sobre el proceso lector y, por tanto, pueden tener mayor capacidad de predicción del mismo. Entre los factores lingüísticos se han considerado las siguientes variables: el nivel de procesamiento fonológico (en adelante PF), el nivel de lectura de letras (LL), el nivel de lectura de las palabras (LP) y nivel de lectura de las pseudopalabras (LPP), la velocidad en denominación de imágenes (VDI), de colores (VDC) y de números (VDN). Como factores no lingüísticos hemos analizado el contexto de instrucción: método de lectoescritura, actividades de aula y materiales de enseñanza.

Para dar respuesta a estas cuestiones, los objetivos científicos de esta investigación se concretan en:

1. Identificar los factores lingüísticos que requieren más atención en la alfabetización temprana y determinar qué variable predice mejor la capacidad lectora del alumnado en edades tempranas.

2. Comparar los niveles de PF, VD y LL, LP y LPP de los centros de la muestra.

3. Determinar la influencia del contexto de instrucción que proporciona el centro (la elección del método de lectura, los recursos y las actividades didácticas empleadas en el aula) así como el nivel de procesamiento fonológico, denominación y lectura de letras, palabras y pseudopalabras del alumnado.

Esta investigación se ha llevado a cabo a través de una metodología mixta que toma en consideración un enfoque cuantitativo y otro cualitativo. De una parte, el enfoque cuantitativo se ha concretado a partir de una metodología predictiva, en la que la lectura de palabras es la variable criterio y las variables predictoras son PF, LL, LPP y VD. Asimismo, se ha realizado un análisis descriptivo para conocer el nivel de PF, LL, LP y LPP de los participantes en el segundo ciclo de Educación Infantil. De otra parte, hemos llevado a cabo un estudio no experimental explicativo para determinar las posibles diferencias entre los centros de la muestra. Las variables dependientes fueron PF, LL, LP, LPP y VD y las variables independientes el centro, la edad y el sexo.

El enfoque cualitativo sobre el contexto de aula se ha concretado a través de dos estudios de casos, cada uno de ellos referidos a dos centros diferentes. En cada centro se han realizado observaciones en el aula y se ha entrevistado al profesorado, teniendo en cuenta los métodos y recursos didácticos utilizados.

\subsection{Población y muestra}

Uno de los puntos de partida de este trabajo establece que el método de lectura utilizado en los centros puede influir en los resultados obtenidos por el alumnado en la lectura temprana. Para poder comprobar esta hipótesis hemos seleccionado al alumnado de Educación Infantil de dos centros de la provincia de Sevilla que difieren en cuanto al método de lectura utilizado, la titularidad, el tamaño y los recursos didácticos. Con respecto al método de lectura, el Centro 1 utiliza un método ascendente alfabético mientras que el Centro 2 utiliza un método mixto fónico-global. Con respecto a otras características, un centro es de titularidad pública (en adelante, Centro 1) y cuenta con 25 alumnos, de los cuales 15 son niños y 10 son niñas; y el otro es de titularidad privada (en adelante, Centro 2) y tiene 80 alumnos, de los cuales 40 son niños y 40 son niñas. La edad de los participantes en ambos centros se encuentra entre los 72 y 83 meses. El Centro 1 se ha incorporado actualmente a la lista de colegios TICS y bilingües de la ciudad; se encuentra situado en una zona de clase media y recibe familias pertenecientes a distintos sectores socioeconómicos y culturales, si bien con predominio de los niveles medio y bajo. En relación con el profesorado, participaron dos maestras de este centro. En el caso del Centro 2, también bilingüe, se encuentra situado en el casco histórico de la ciudad recibiendo a un alumnado de familias pertenecientes a un contexto socioeconómico de clase alta. En este centro se contó con la participación de otras dos maestras. 


\subsection{Instrumento de recogida de datos}

Para medir las variables del estudio (PF, VD y LL, LP y LPP) se ha usado la primera sección (preparación a la lectura y escritura) de la prueba ALE1 (Actividades para el aprendizaje de la lectura y la escritura) de González y Cuetos (2008). En esta prueba, el alumnado debía realizar actividades de identificación de sonidos en palabras; denominación de objetos, colores y números; y leer letras, palabras y pseudopalabras. La fiabilidad fue calculada en septiembre de 2017 con el alumnado de la muestra participante en este estudio mediante el coeficiente Alfa de Cronbach y su valor fue de 0,758.

En el contexto aula, el estudio de la lectura recoge datos cualitativos. Mediante la observación participante se han recogido notas de campo sobre el método de lectura y los recursos utilizados en clase. Las observaciones se realizaron todos los jueves entre el 23 de marzo y el 25 de mayo de 2017, con un total de 38,5 horas. Asimismo, a través de entrevistas semiestructuradas con el profesorado se ha recogido información sobre su formación pedagógica, el material y el método de lectura que utilizan en clase. En dichas entrevistas se preguntaron cuestiones como: ¿Qué tipo de material usa? ¿auténtico, adaptado, original, libros, internet...? ¿Qué editorial? ¿Considera que el material de clase es apropiado para la enseñanza que realiza? ¿Qué método de lectoescritura utiliza? (ascendente (fónico, silábico, alfabético) o descendente (global, léxico o integral)) ¿Cuántas horas dedica a las prácticas de lectura en clase?

\subsection{Técnicas de análisis}

En lo que se refiere al análisis de los datos cuantitativos, primero se llevó a cabo un análisis de regresión lineal usando el método paso a paso (stepwise), para continuar con el cálculo de estadísticos descriptivos como la media y la desviación típica para los resultados de la prueba ALE1. Posteriormente, se analizaron las diferencias para el conjunto de las pruebas PF, LL, LP, LPP y VD en función de los centros de la muestra, el sexo y la edad del alumnado, mediante la prueba t de Student para grupos independientes. En el caso de los datos de naturaleza cualitativa, se ha realizado un análisis de contenido de las observaciones en el aula y de las entrevistas de los profesores.

\section{Resultados}

\subsection{Predicción de la capacidad lectora}

Los resultados obtenidos al analizar las relaciones entre las diferentes pruebas muestran correlaciones altas entre la lectura de palabras y pseudopalabras $(\mathrm{rXY}=0,867)$, la lectura de palabras y letras $(\mathrm{rXY}=0,709)$, la lectura de palabras y el procesamiento fonológico $(\mathrm{rXY}=0,670)$. En cambio, la correlación de las puntuaciones en lectura de palabras con las denominaciones de imágenes, colores y números es más baja (p.e. para números, $\mathrm{rXY}=0,321$ ) o próxima a cero. Estos resultados permitirían tomar en consideración la capacidad predictiva de las variables lectura de letras, pseudopalabras, procesamiento fonológico y denominaciones sobre la lectura de palabras.

Los datos obtenidos en la ecuación de regresión indican que la conciencia fonológica, la lectura de letras y pseudopalabras, así como la velocidad en la denominación de objetos (vocabulario en imágenes) explicarían las diferencias halladas en la lectura de palabras en los participantes de la muestra.

La ecuación que resulta del análisis de regresión explica el 79,2\% de la varianza observada (R2 ajustado) en la lectura de palabras; cabe mencionar la contribución de la LPP $(B=0,620$, y p $=0,0001)$ y el escaso poder predictivo de la velocidad de denominación de imágenes (DI). En cambio, la velocidad en denominación de colores y números quedan excluidos de la de la ecuación final. Como puede verse en la Tabla 1, es posible diferenciar de forma estadísticamente significativa entre la varianza debida a dichas variables y a la varianza de error $(\mathrm{p}<0,05)$. 
Tabla 1

Coeficientes de la ecuación de regresión

\begin{tabular}{|c|c|c|c|c|c|}
\hline \multirow[t]{2}{*}{ Modelo } & \multicolumn{2}{|c|}{$\begin{array}{l}\text { Coeficientes no } \\
\text { estandarizados }\end{array}$} & \multirow{2}{*}{$\begin{array}{c}\text { Coeficientes } \\
\text { estandarizados }\end{array}$} & \multirow[t]{2}{*}{$\mathbf{t}$} & \multirow[t]{2}{*}{ Sig. } \\
\hline & B & Error estándar & & & \\
\hline Constante & .618 & 1.13 & & .542 & .589 \\
\hline Lectura de pseudopalabras & .620 & .07 & .612 & 8.670 & .000 \\
\hline Lectura de letras & .034 & .01 & .157 & 2.415 & .018 \\
\hline Procesamiento fonológico & .048 & .01 & .232 & 3.238 & .002 \\
\hline $\begin{array}{l}\text { Denominación de imágenes } \\
\text { (vocabulario) }\end{array}$ & -.086 & .03 & -.125 & -2.365 & .020 \\
\hline
\end{tabular}

4.2. Grado de dificultad en el procesamiento fonológico, lectura y denominación

Los resultados obtenidos por el alumnado de la muestra indican que las pruebas de denominación de imágenes (DI), colores (DC) y números (DN) fueron las más fáciles de realizar por parte del alumnado participante. Así, como puede verse en la

Tabla 2, las puntuaciones medias (M) e incluso el percentil 25 (P25) están muy próximos al valor máximo (puntuación 36) en dichas pruebas. Por el contrario, la prueba lectura de pseudopalabras es la más difícil con una media que representa el $64 \%$ de la puntuación máxima, seguida de la lectura de palabras (la media es un $68 \%$ del valor máximo), el procesamiento fonológico (media de $82 \%$ sobre el máximo) y la lectura de letras (media $87 \%$ sobre el máximo).

Tabla 2

Estadísticos descriptivos correspondientes a las pruebas

\begin{tabular}{cccccccc}
\hline & \multicolumn{3}{c}{ Denominación } & & Lectura & \\
\hline & Imagen & Color & Número & Letra & Palabra & $\begin{array}{c}\text { Pseudo- } \\
\text { palabra }\end{array}$ & $\begin{array}{c}\text { Proc. } \\
\text { fonológico }\end{array}$ \\
M. & 34.81 & 34.97 & 35.56 & 31.42 & 3.42 & 3.19 & 57.94 \\
D. T & 2.76 & 3.33 & 1.14 & 8.82 & 1.90 & 1.88 & 9.25 \\
Mín. & 21 & 13 & 28 & 1 & 0 & 0 & 27.00 \\
Máx. & 36 & 36 & 36 & 36 & 5 & 5 & 71.00 \\
P25 & 35.00 & 35.00 & 36.00 & 32.00 & 2.00 & 1.00 & 51.75 \\
P50 & 36.00 & 36.00 & 36.00 & 36.00 & 4.00 & 4.00 & 60.00 \\
P75 & 36.00 & 36.00 & 36.00 & 36.00 & 5.00 & 5.00 & 65.00 \\
\hline
\end{tabular}

M. $=$ Media; DT $=$ Desviación Típica; P.=Percentil.

\subsection{Diferencias en función del centro}

$\mathrm{El}$ análisis realizado muestra que las respuestas del alumnado a la prueba ALE1 no arrojan diferencias estadísticamente significativas $(\mathrm{p}<0,05)$ para las variables sexo y edad. Con respecto al sexo, las desviaciones típicas de ambos grupos son similares con la excepción de la prueba en denominación de colores en la que las niñas obtuvieron puntuaciones más altas y menos dispersas $(\mathrm{M}=35,69 ; \mathrm{DT}=0,70)$ que las de los niños $(\mathrm{M}=34,17 ; \mathrm{DT}=4,7)$. En cuanto a la edad, las medias de los discentes de menor edad ( 72 a 76 meses) tienden a ser inferiores que las de sus compañeros de mayor edad (77 a 83 meses). El resultado fue similar cuando se consideraron tres grupos de edad (74, 77 y 79 meses, respectivamente). No obstante, dichas diferencias no resultaron estadísticamente significativas.

La Tabla 3 recoge las medias y desviaciones típicas de las puntuaciones de los participantes en las pruebas en función del centro en el que estudian. En todas las pruebas relativas a la lectura de letras, palabras y pseudopalabras, así como en el procesamiento fonológico, se encontraron diferencias estadísticamente significativas entre el alumnado de los dos centros. Las medias más altas 
siempre corresponden al alumnado del Centro 2, al igual que las desviaciones típicas más bajas (excepto en la lectura de pseudopalabras); esto vendría a indicar que en el Centro 2 el nivel lector de los discentes no sólo es más alto sino también más uniforme que en el Centro 1.

Tabla 3

Medias y desviación estándar en función del centro

\begin{tabular}{lcccc}
\hline & Colegio & N & M. & D.T. \\
\hline Lectura de letras & Centro 1 & 25 & 22.92 & 13.03 \\
& Centro 2 & 77 & $34.18^{* *}$ & 4.28 \\
Lectura de palabras & Centro 1 & 25 & 1.04 & 1.39 \\
& Centro 2 & 77 & $4.19^{* *}$ & 1.31 \\
Lectura de pseudopalabras & Centro 1 & 25 & 1.04 & 1.27 \\
& Centro 2 & 77 & $3.88^{* *}$ & 1.47 \\
Procesamiento fonológico & Centro 1 & 25 & 46.92 & 7.84 \\
& Centro 2 & 77 & $61.51 * *$ & 6.42 \\
Denominación de imágenes & Centro 1 & 25 & 35.20 & 2.73 \\
& Centro 2 & 77 & 34.69 & 2.77 \\
Denominación de colores & Centro 1 & 25 & 34.76 & 4.57 \\
& Centro 2 & 77 & 35.04 & 2.85 \\
Denominación de números & Centro 1 & 25 & 35.28 & 1.40 \\
& Centro 2 & 77 & 35.65 & 1.04 \\
\hline
\end{tabular}

Nota: ${ }^{* *} \mathrm{p}<.001$

El análisis de las correlaciones entre las puntuaciones de las pruebas obtenidas por el alumnado en cada uno de los centros también muestra valores diferentes. Así, en el Centro 1, las correlaciones entre las variables procesamiento fonológico y la lectura de letras, palabras y pseudopalabras son estadísticamente significativas $(\mathrm{p}<0,01)$ e iguales o superiores a 0,60 . Por el contrario, en el Centro 2 , para las mismas variables, las correlaciones no alcanzan 0,30 y sólo dos de ellas son estadísticamente significativas.

En el centro 1, los valores de las altas correlaciones obtenidas entre las puntuaciones de las pruebas ( $\mathrm{r}$ PF, $\mathrm{LL}=0,626 ; \mathrm{r} \mathrm{PF}, \mathrm{LP}=0,661 ; \mathrm{r} \mathrm{PF}, \mathrm{LPP}=0,592)$ ) pueden explicarse porque dicho alumnado tiene un elevado nivel de dependencia del procesamiento fonológico al leer palabras y pseudopalabras a través de una ruta subléxica. La alta correlación existente entre el procesamiento fonológico y las letras podría reflejar la influencia del método de lectura utilizado.

En el centro 2, los valores de las correlaciones obtenidas entre las puntuaciones de las pruebas ( $\mathrm{r}$ PF, $\mathrm{LL}=0,065 ; \mathrm{r}$ PF, $\mathrm{LP}=0,232 ; \mathrm{r} \mathrm{PF}, \mathrm{LPP}=0,293$ ) puede explicarse porque los discentes a medida que mejoran su capacidad de lectura cambian de una ruta subléxica a una ruta léxica y, en consecuencia, tienen una menor dependencia del procesamiento fonológico. En este caso, la casi nula correlación existente entre el procesamiento fonológico y la lectura de letras podría reflejar la influencia de un método de lectura que sitúa a los discentes en un momento de desarrollo lector más avanzado.

El análisis de las observaciones realizadas en el centro, así como la recogida de datos de naturaleza contextual nos han permitido realizar una explicación tentativa de las diferencias (Tabla 4). Así, el centro que obtiene los resultados más bajos, el Centro 1, también presenta unas características educativas que lo sitúan en desventaja respecto al Centro 2. (Ver Tabla 4).

En relación a los recursos de los que disponen los centros, las aulas del Centro 1 cuentan con libros de lectura que se caracterizan por la inclusión de dibujos y frases simples proporcionadas por una editorial de libros de lectura. El material colgado en la pared incluye números, días de la semana, el abecedario en mayúsculas y minúsculas con dibujos identificativos. La gran mayoría de estos materiales están al alcance de los niños. En este centro se usan libros de una editorial comercial y fotocopias de otros materiales publicados. En el aula, durante las observaciones realizadas, no se utilizaron materiales audiovisuales ni internet. 
Las actividades que se realizan en clase del Centro 1 se basan en las establecidas en el currículo. El método de lectura utilizado es un método ascendente alfabético. En una sesión típica, la profesora comienza presentando la denominación de una letra a los discentes, que la repiten, para seguir uniendo a la misma las vocales. Por tanto, los contenidos se presentan de forma aislada sin contar con un contexto lingüístico complementario (cuento, lectura) que les ayude a alcanzar un aprendizaje más significativo desde el punto de vista de la comunicación.

En la entrevista realizada a las profesoras, el uso de un método alfabético se justificaba por su falta de formación en otros métodos de lectura, como por ejemplo, el conocimiento de un método fónico. Podemos ver su interés por este tipo de métodos en la siguiente cita textual:

La logopeda nos dio un curso y nos proporcionó muchas cosas para niños con problemas, y lecturas. Es verdad que el niño dice "be" pero el sonido no es ese. Nos dijo que no, que no le enseñáramos el nombre de la letra, sino el sonido. No estaba mal, pero tiene su razón de ser. Si aprende el nombre de la letra, lee mal. Si el niño aprende el sonido con un gesto lo adquiere mejor (...). Yo lo voy a probar este año porque me ha convencido (...) (Entrevista profesora 1 del Centro 1)

Con respecto a la interacción observada en el aula la que se produce de forma más frecuente es la interacción entre la profesora y el alumnado, no registrándose ejemplos de actividades de lectura y escritura compartida entre el alumnado.

Por otra parte, en lo que respecta a los recursos, las aulas del Centro 2 disponen de tarjetas colgadas en el aula que se centran en números, días de la semana, abecedario en mayúsculas y minúsculas con dibujos identificativos y palabras, que se encuentran al alcance de los niños. En este centro se usa material propio del colegio adaptado y adecuado a los intereses y necesidades del alumnado, haciendo uso de páginas web y blogs de las asignaturas.

Las actividades que se realizan en clase están basadas en el aprendizaje de la conciencia fonológica, la fluidez lectora, el vocabulario y la comprensión. Dado que se trata de alumnos que se encuentran en una etapa inicial de lectura, buena parte de las actividades que se llevan a cabo forman parte de una lectura compartida de palabras (entre el maestro, como lector experto, y el discente). Una de las profesoras señala: “(...) mi objetivo final: conseguir que todos mis niños lean, intento equilibrar los diferentes componentes de la lectura, la conciencia fonémica, la fonética, la fluidez, el vocabulario y la comprensión en la enseñanza diaria" (Entrevista profesora 1 del Centro 2).

El método de lectura utilizado es un método ascendente fónico combinado con actividades propias del método global. La profesora comienza cada sesión presentando el sonido de una letra a través de juegos que incluyen actividades como el ordenamiento de letras con ilustraciones, el deletreo de palabras, juegos para identificar objetos que empiecen con una letra y el encadenadenamiento de palabras según la sílaba. Además, hace indicaciones del material colgado que muestran palabras familiares referidas a los objetos del aula. Las lecturas están precedidas por una introducción que sitúa al alumnado en el contexto de las historias que se van a trabajar (Swartz, 2018). Una sesión típica se desarrolla a partir de historias y anécdotas personales que se relacionan con la experiencia real. Por ejemplo, la profesora cuenta una historia personal, relacionada con la compra en un supermercado, a partir de la cuál extrae palabras que asocia a dibujos, procediendo a realizar ejercicios de deletreo, segmentación y reconocimiento de sonidos. El contexto de la historia y dibujos ayuda a los niños a comprender el significado de las palabras que se trabajan a través del método.

Finalmente, en lo que respecta a la interacción se observan varios tipos de relaciones, por un lado de la profesora hacia el alumnado, y por otro, entre los mismos discentes tanto por parejas como en pequeños grupos. 
Tabla 4

Matriz cruzada de los centros

\begin{tabular}{lll}
\hline & \multicolumn{1}{c}{ Centro 1 } & \multicolumn{1}{c}{ Centro 2 } \\
\hline Método de enseñanza & $\begin{array}{l}\text { Método ascendente: alfabético, } \\
\text { complementado con actividades de } \\
\text { deletreo y sonidos. }\end{array}$ & $\begin{array}{l}\text { Método ascendente: fónico, } \\
\text { complementado con actividades } \\
\text { del método global. }\end{array}$ \\
Materiales didácticos & $\begin{array}{l}\text { Libros de una editorial comercial y } \\
\text { fichas. }\end{array}$ & $\begin{array}{l}\text { Material elaborado por el } \\
\text { colegio y adaptado a cada } \\
\text { discente. }\end{array}$ \\
Actividades & Basadas en el currículo y material. & $\begin{array}{l}\text { Basadas en experiencias reales y } \\
\text { adaptadas al alumnado. }\end{array}$ \\
Interacciones & Profesorado-alumnado & $\begin{array}{l}\text { Profesorado-alumnado y } \\
\text { alumnado-alumnado }\end{array}$ \\
\hline
\end{tabular}

\section{Discusión de resultados}

El proceso de adquisición de la lectura es un proceso complejo que, tal y como indica la literatura especializada, necesita una instrucción específica (Márquez, 2013; Ehri, 1987; De-La-Calle Cabrera, 2017). Este trabajo comienza determinando qué factores lingüísticos tienen una mayor incidencia en el proceso lector y, por ende, aquellos cuyo desarrollo resulta conveniente analizar y potenciar para mejorar la enseñanza de la lectura en edades tempranas.

Con relación al primer objetivo, el análisis de regresión realizado muestra que el procesamiento fonológico, la lectura de letras y pseudopalabras son los factores determinantes de la lectura de palabras. De hecho, PF, LL y LPP predicen casi el $80 \%$ de la variabilidad observada en la lectura de palabras. En cambio, la velocidad en denominación de imágenes, colores o números tienen una escasa capacidad explicativa de dicha variable. El análisis de la dificultad de las pruebas de PF, LL, LPP y VD para el alumnado indica que las pruebas PF, LL y LPP presentan también un mayor nivel de dificultad que las pruebas relacionadas con la VD. En este sentido, las pruebas de VD tienen una menor capacidad de discriminación entre los discentes, lo que explicaría el escaso poder predictivo de este tipo de pruebas; por el contrario, la dificultad que muestran las pruebas de PF, LL y LPP permite recoger diferencias entre el alumnado, lo que las convierten en buenas predictoras de la lectura de palabras. Nuestros resultados confirman los estudios que señalan que existen altas correlaciones entre el procesamiento fonológico y la lectura de letras, palabras y pseudopalabras (Edyburn et al., 2017; Hill, 2017; Jiménez et al., 2000; Jiménez et al., 2007; Jiménez et al., 1995). Además, nuestros resultados también coinciden con los obtenidos en estudios predictivos sobre la lectura como los de Ehri (1987); Batson-Magnuson (2017); Saracho (2017); Biscoe Kenner et al. (2017); Bellocchi et al. (2017); Suárez-Coalla et al. (2013); Schaars et al. (2017); Parrilla et al. (2004).

Con relación al segundo objetivo planteado en nuestro estudio, los resultados obtenidos en las pruebas PF, LL, LP y LPP por el alumnado de ambos centros permiten afirmar que existen diferencias estadísticamente significativas a favor de los participantes del Centro 2. Estas diferencias no se pueden atribuir a variables individuales en función de la edad o el sexo, dado que el análisis de estas variables muestra que no existen diferencias estadísticamente significativas entre el alumnado de 72 y 83 meses de edad ni en función de su sexo. El análisis comparativo de los resultados en ambos centros se complementa con el análisis de las correlaciones entre el procesamiento fonológico y la lectura de palabras en el que podemos ver que el alumnado del Centro 2, a pesar de presentar resultados más altos en el procesamiento fonológico, tiene correlaciones más bajas con la lectura de palabras que las que encontramos en el Centro 1. Esto podría deberse a que el alumnado del Centro 2 se encuentre haciendo uso de una ruta léxica en la que se ha llegado a la automatización de todos los sonidos y su almacenamiento léxico comienza a funcionar; por el contrario, el alumnado del 
Centro 1 siguen utilizando únicamente una ruta subléxica. Coincidimos así con estudios como los de Frith (1986); Guzmán-Simón, Navarro-Pablo y García-Jiménez (2015); Coltheart (1985); Cuetos (2008), quienes confirman la diferenciación del grado de automatización del contenido lingüístico. En este sentido, el centro 2 demuestra un grado más avanzado de automatización al encontrarse en una ruta léxica. Esto también les permite conectar la lectura con la comprensión de lo que leen. Así entendemos que esto, además, puede deberse al esfuerzo e interés que nos manifiesta su profesora en la entrevista y sitúa la comprensión lectora en un foco de interés en el aprendizaje dándole la importancia que le conceden autores como Esteban Peregrina (2017) o Swartz (2018).

El tercer objetivo de nuestro estudio se centra en explicar las diferencias del alumnado en la lectura de palabras y su influencia en el contexto de instrucción que proporciona cada centro. El hallazgo más importante encontrado es que el método de lectura influye en el nivel lingüístico de los discentes. La combinación, fónico con global, que utiliza el Centro 2 resulta ser mucho más efectiva que el uso del método alfabético del Centro 1, dado que el alumnado del Centro 2, además de obtener mejores resultados en la prueba de lectura, demuestra encontrarse en un nivel de desarrollo lector superior. Coincidimos con Cuetos (2008), Jiménez et al. (2007), Schoaga et al. (2017) y Jiménez et al. (2001) en que la utilización de un método fónico favorece el desarrollo de la conciencia fonológica y, por tanto, de la lectura.

En relación a estos resultados, cabe destacar también que las altas puntuaciones obtenidas por el Centro 2 también podrían estar relacionadas con el tipo de actividades que realiza el profesorado en el aula, elaboradas por el profesorado del centro y adaptadas a las necesidades del alumnado. Dichas actividades potencian el desarrollo de habilidades lectoras a través de imágenes visuales que permiten el reconocimiento de elementos familiares para el discente en juegos, canciones y, sobre todo, mediante las interacciones que son más variadas que en el centro 1 (profesorado-alumnado, alumnado-alumnados). Así, coincidimos con Medina, Valdivia y San Martín (2014) en la importancia de la interacción en el aula y su posible efecto positivo en el aprendizaje.

Además, las actividades están basadas en la experiencia real, es decir, actividades que potencien las relaciones diarias de la vida real a través de un clima positivo en el contexto del aula. De este modo, coincidimos con autores como Beecher et al. (2002), Verhoeven (2005), Bigas (2002) Norling et al. (2015), Englezou et al. (2014) Fons (2002) Montessori (2006), Freinet (1974), Teberosky (2003) y Ferreiro (2002) que apoyan el uso de actividades que potencien la experiencia real en aula. De esta manera, no solo se desarrolla la motivación del alumnado por el conocimiento del mundo real, sino que se desarrollan habilidades comunicativas y lingüísticas de una manera eficaz y dinámica.

Estos resultados deben interpretarse a la luz de las variables que hemos considerado en este estudio, aunque podrían considerarse otras como el capital cultural que tienen las familias de los niños; es decir, las ventajas culturales que las familias poseen (habilidades, conocimientos, actitudes y otros recursos culturales) y que pueden beneficiarlas dentro de un contexto social concreto (Miller, Hilgendorf \& Dilworth-Bart, 2014; Bourdieu, 1986).

\section{Conclusiones y prospectiva}

Teniendo en cuenta los tres objetivos planteados en este estudio, concluimos que el procesamiento fonológico, la lectura de letras y pseudopalabras son los factores determinantes de la lectura de palabras. Estas variables se muestran como los mejores predictores de la lectura de palabras. Las diferencias entre centros no se pueden explicar a partir de las características individuales de sexo o edad, sino a partir del método de lectura utilizado en el aula. Otras variables, como el capital cultural, podrían ser objeto de estudio en próximos trabajos.

Los niveles de PF, LL, LP y LPP que presentan los discentes del Centro 2 son más altos y las correlaciones entre PF y LP son más bajas. Una posible explicación es que el alumnado del Centro 2 utiliza la ruta léxica en la que se produce la automatización de todos los sonidos. Este centro ha estado implementando un método fónico combinado con global a lo largo del ciclo de Educación Infantil, además de actividades elaboradas por el propio profesorado, basadas en la experiencia real, adaptadas al alumnado y que potencian distintos tipos de interacciones en el aula. La combinación del método 
con dichas actividades ha permitido que el alumnado del Centro 2 alcance un mayor desarrollo de conciencia fonológica y, por tanto, de sus habilidades de lectura.

Finalmente, los centros seleccionados para el estudio son bilingües porque este trabajo tendrá una proyección en un estudio posterior basado en el análisis de la segunda lengua y los métodos de enseñanza. Partimos de la idea de que cuando se introduce el aprendizaje de la lectura en una segunda lengua en estas edades es conveniente tener en cuenta el método que se está utilizando para la lectura en la primera lengua de instrucción. Los resultados obtenidos en este trabajo que muestran los beneficios de la utilización de un método fónico abren las puertas a la continuidad del estudio con la implementación de un método fónico para el aprendizaje de la segunda lengua, tal y como proponen autores como Lázaro (2007) y López-Cirugeda y López-Campillo (2016).

\section{Referencias bibliográficas}

Batson-Magnuson, L. (2017). An Analysis of the Relationship Between Phonological and Nonphonological Language and Early Reading Development. Communication Disorders Quarterly, 38 (2), 78-88. doi: $10.1177 / 1525740116644891$

Beecher, B., y Makin, L. (2002). Languages and literacies in early Childhood: At home and in early childhood settings. European Early Childhood Education Research Journal, 10 (1), 67-84. doi: $10.1080 / 13502930285208851$

Bellocchi, S., Tobia, V. y Bonifacci, P. (2017). Predictors of Reading and comprehension abilities in bilingual and monolingual children: a longitudinal study on a transparent language. Reading and Writing, 30, 1311-1334. doi: 10.1007/s11145-017-9725-5

Bigas, M. y Correig, M. (2002). Didáctica de la Lengua en la Educación Infantil. Madrid, España: Síntesis Educación.

Bigas, M. (2002). El lenguaje oral en la escuela infantil. En Bigas, M. y Correig, M. Didáctica de la Lengua en la Educación Infantil. (pp. 43-70). Madrid, España: Síntesis Educación.

Biscoe Kenner, B., Patton Terry, N., Friehling, A. H. y Namy, L. L. (2017). Phonemic awareness development in 2.5- and 3.5-year-old children: an examination of emergent, receptive, knowledge and skills. Reading and Writing, 30, 1575-1594. doi: 10.1007/s11145-017-9738-0

Bourdieu, P. (1986) The Forms of Capital, in J.G. Richardson (Ed.) Handbook of Theory and Research for the Sociology of Education, pp. 241-258. New York: Greenwood Press.

Casillas, A. y Goikoetxea, E. (2007). Sílaba, principio-rima y fonema como predictores de la lectura y la escritura tempranas. Infancia y Aprendiraje, 30 (2), 245-259. doi: 10.1174/021037007780705184

Coltheart, M. (1985). Cognitive neuropsychology and the study of Reading. En M. Posner y G. Marin (Eds.). Attention and performance XL. Hillsdale: LEA.

Cuetos, F. (2008). Psicología de la lectura. Madrid, España: Wolters Kluwer Educación.

De-La-Calle Cabrera, A. M. (2017). Por una Intervención Educativa Pertinente en la Alfabetización Temprana: Desarrollo de un Programa Piloto con Niños Españoles de 3 y 4 Años. Porta Linguarum, Monográfico II, 27-41.

Edyburn, K. L., Quirk, M., Feliz, E., Swami, S., Goldstein, A., Terzieva, A. y Scheller, J. (2017). Literacy Screening Among Latino/a and Dual Language Learner Kindergarteners: Predicting First Grade Reading Achievement. Literacy Research and Instruction, 56 (3), 250-267. doi: 10.1080/19388071.2017.1305470

Ehri, L.C. (1987). Movement in word reading and spelling: How spelling contributes to reading. Center for the Study of Reading. Technical Reports, 408. Illinois: University of Illinois at UrbanaChampaign Library.

Englezou, E., y Fragkouli, E. (2014). Literacy in the early years and English as an additional language: The case of a British international school. Journal of Research in International Education, 13 (1), 46-62. doi: $\underline{10.1177 / 1475240914528851}$ 
Esteban Peregrina, A. (2017). Estado actual de la comprensión lectora en Educación Primaria. Revista Fuentes, 19 (1), 15-37. doi: 10.12795/revistafuentes.2017.19.1.01

Ferreiro, E. (2002). Alfabetización: teoría y práctica (5a ed.). México DF, México: Siglo XXI

Fons, M. (2002). Aprender a leer y a escribir. En Bigas, M. y Correig M. Didáctica de la Lengua en la Educación Infantil. (pp. 157-178). Madrid, España: Síntesis Educación.

Freinet, C. (1974). El método natural de lectura. Barcelona: Laia.

Frith, U. (1986). A Developmental Framework for Developmental Dyslexia. Annals of Dyslexia, 36, 69-81.

González, R. M. y Cuetos, F. (2008). ALE1: Actividades para el aprendiraje de la lectura y la escritura. Cuaderno de evaluación inicial. Madrid, España: CEPE.

Guzmán-Simón, F. Navarro-Pablo, M. y García-Jiménez, E. (2015). Escritura y lectura en Educación Infantil. Conceptos, secuencias didácticas y evaluación. Manual. Madrid, España: Ediciones Pirámides.

Hill, S. (2017). Early reading and practice-inspired research. Early Child Development and Care, 187 (34), 322-330. doi: $10.1080 / 03004430.2016 .1234468$

Jiménez, J. E. y Artiles, C. (2001). Cómo preveniry corregir las dificultades en el aprendizaje de la lectoescritura. Madrid, España: Síntesis.

Jiménez, J. E. y Ortiz, M. R. (2000). Metalinguistic Awareness and Reading Acquisition in the Spanish Language. The Spanish Journal of Psychology, 3 (1), pp. 37-46.

Jiménez, J. E. y Ortiz, M.R. (2007). Conciencia Fonológica y aprendizaje de la lectura: Teoría, evaluación e intervención. Madrid, España: Síntesis.

Jiménez, J. E. y Haro, C. R. (1995). Effects of Word Linguistic Properties on Phonological Awareness in Spanish Children. Journal of Educational Psychology, 87 (2), 193-201. doi: 10.1037//00220663.87.2.193

Lázaro Ibarrola, A. (2007). Enseñanza de la lectura a través de phonics en el aula de Lengua Extranjera en Educación Primaria. Porta Linguarum, 8, 153-167.

Lera Rodríguez, M. J. (2007). Calidad de la Educación Infantil: instrumentos de evaluación. Revista de Educación, 343, 301-323.

López-Cirugeda, I. y López-Campillo, R.M. (2016). El método sistemático-sintético de lectoescritura Phonics como herramienta para la adquisición de la fonética inglesa. Revista Fuentes, 18 (2), 183-195. doi: $10.12795 /$ revistafuentes.2016.18.2.05

Márquez, M. (2013). Estrategias utilizadas en aulas bilingües en la enseñanza de la lectura en español. Tesis doctoral. Universidad de Texas-Pan American. Recuperado de http://0search.proquest.com.fama.us.es/index (Febrero de 2017)

Medina, L., Valdivia, A. y San Martín, E. (2014). Prácticas Pedagógicas Para la Enseñanza de la Lectura Inicial: Un Estudio en el Contexto de la Evaluación Docente Chilena. PSYKHE, 23 (2), 1-13. doi: $10.7764 /$ psykhe.23.2.734

McClelland, JL. (1987). The case for interactionism in language processing. En M. Coltheart (Ed), The Psychology of Reading. Hoye: LEA.

Miller, K., Hilgendorf, A., \& Dilworth-Bart, J. (2014). Cultural Capital and Home-School Connections in Early Childhood. Contemporary Issues in Early Childhood, 15 (4). doi: 10.2304/ciec.2014.15.4.329

Montessori, M. (2006). Ideas generales sobre el método. Manual práctico. Ed. 2. Madrid: CEPE.

Norling, M., Sandberg, A., y Almqvist, L. (2015). Engagement and emergent literacy practices in Swedish preschools. European Early Childhood Education Research Journal, 23 (5), 619-634. doi: 10.1080/1350293X.2014.996423

Parrilla, R., Kirby, J. R. y McQuarrie, L. (2004). Articulation rate, naming speed, verbal short-term memory and phonological awareness: Longitudinal predictors of early Reading development? Scientific Studies of Reading, 8 (1), 3-26. doi: 10.1207/s1532799xssr0801 2

Pinto, G., Bigozzi, L., Vezzani, C., y Tarchi, C. (2016). Emergent literacy and reading acquisition: a longitudinal study from kindergarten to primary school. European Journal of Psychology of Education, 1-17. doi: 10.1007/s10212-016-0314-9 
24 Sara Isabel Rendón Romero, Eduardo García Jiménez \& Macarena Navarro Pablo

Saracho, O. N. (2017) Literacy and language: new developments in research, theory, and practice. Early Child Development and Care, 187 (3)-4, 299-304. doi: 10.1080/03004430.2017.1282235

Schaars, M. M. H., Segers, E. y Verhoeven, L. (2017). Word decoding development in incremental phonics instruction in a transparent orthography. Reading and Writing, 30, 1529-1550. doi: $10.1007 / \mathrm{s} 11145-017-9735-3$

Schoaga, O., Akintola, O. A. y Isiwat OKPOR, C. (2017). Nurturing Reading Proficiency of Pupils through Phonics: Entrepreneurial Opportunities for Early Childhood Educators in Nigeria. Journal of Education and Practice, 8 (11), 103-108.

Swartz, S. (2018). Estrategias innovadoras para enseñar a leer y escribir. Cada niño un lector. México: Gil Editores.

Suárez-Coalla, P. García-de-Castro, M. y Cuetos, F. (2013). Variables predictoras de la lectura y la escritura en castellano. Infancia y Aprendizaje, 36 (1), 77-89. doi: 10.1174/021037013804826537

Teberosky, A. (2003). Propuesta constructivista para aprender a leer y a escribir. Barcelona, España: Vicens Vives.

Verhoeven, L. (2005). Literacy development across language boundaries. En Ravid, D.D. Shyldkrot, H.b. Perspectives on Language and Language Development: essays in honor of Ruth A. Berman. (pp. 437452). USA: Kluwer Academic publishers.

\section{Para citar este artículo}

Rendón Romero, S.I., García Jiménez, E:, \& Navarro Pablo, E. (2019). La lectura de palabras: la influencia del procesamiento fonológico y del método lectoescritor. Revista Fuentes, 21(1), 11 24. [Fecha de consulta: dd/mm/aa]. doi: 10.12795/revistafuentes.2019.v21.i1.01 